\title{
Group B Streptococcal Colonization in 160 Mother-Baby Pairs: A Prospective Cohort Study
}

\author{
Alberto Berardi, $\mathrm{MD}^{1}$, Cecilia Rossi, $\mathrm{MD}^{1}$, Roberta Creti, $\mathrm{PhD}^{2}$, Mariachiara China, $\mathrm{MD}^{3}$, Giovanni Gherardi, $\mathrm{PhD}^{4}$, \\ Claudia Venturelli, $\mathrm{MD}^{5}$, Fabio Rumpianesi, $\mathrm{MD}^{5}$, and Fabrizio Ferrari, $\mathrm{MD}^{1}$
}

Objectives To identify the source of postnatal colonization with group B Streptococcus (GBS) and to evaluate the impact of intrapartum antibiotic prophylaxis (IAP) administration in newborn infant transmission.

Study design A prospective, longitudinal study evaluated GBS colonization in 160 mother-baby pairs. Specimens were collected from the time of delivery to 8 weeks post-partum, from rectum, vagina, and milk of mothers, and from throat and rectum of neonates. Women were grouped according to their GBS status at discharge from the hospital: culture-positive carriers $(n=83)$, culture-negative carriers $(n=26)$, and noncarriers $(n=51)$. Newborns were considered colonized if GBS was yielded from at least 1 site.

Results A total of 35 (21.9\%) neonates were colonized; 30 were born to culture-positive carriers, 2 to culturenegative carriers, and 3 to noncarriers. Infants of culture-positive carriers exposed to IAP were less likely to be colonized (15/57 vs $15 / 26, P=.01)$, or heavily colonized, ( $7 / 57 \mathrm{vs} 9 / 26, P=.04)$. Of all newborns, those exposed to IAP and discharged GBS-free from hospital, often became colonized subsequently (12/57 vs 1/26, $P=.09)$. Molecular typing analysis (available for 30 of 32 carrier mothers and their infants) confirmed an identical strain of GBS in all mother-baby pairs. Six of 83 culture-positive carrier mothers had a positive milk culture. Their respective neonates all were heavily colonized.

Conclusions Newborns exposed to IAP and GBS-free at hospital discharge subsequently acquire GBS from their mothers. Culture-positive milk is associated with heavy neonatal colonization. (J Pediatr 2013;163:1099-104).

roup B Streptococcus (GBS) is the leading cause of neonatal sepsis, pneumonia, and meningitis. GBS infection can occur from birth through day 6 of life (early-onset disease, EOD) or from day 7 through 89 (late-onset disease, LOD).$^{1-3} \mathrm{EOD}$ results from the vertical transmission of GBS (at delivery or shortly before) from a mother who is colonized at the anorectal and vaginal sites. The administration of intrapartum antibiotic prophylaxis (IAP) has dramatically reduced the rates of EOD, but LOD continues to cause morbidity and mortality. ${ }^{1-4}$ Routes of GBS transmission to the newborn infant following hospital discharge are poorly understood, hampering efforts at prevention. Such transmission is a necessary precursor to the development of LOD in some colonized infants.

Maternal GBS colonization is a main risk factor, ${ }^{5}$ and $\sim 50 \%$ of infants with LOD are GBS colonized at birth with the maternal serotype. ${ }^{6}$ Nosocomial transmission of GBS, through the hands of healthcare workers, was frequent some decades ago, when postparturient mothers and their infants typically remained in the hospital $\geq 1$ week. $^{7-9}$ However, neonates "room in" with their mothers, and transmission routes may have changed.

Anecdotal case reports have suggested breast milk as a possible source of LOD. ${ }^{10-12}$ However, the relationship between contaminated milk and LOD is not yet clear. The predominant routes of LOD transmission are poorly understood. Since the introduction of IAP as a standard EOD prevention practice, the neonatal colonization has not been studied in detail. To address these unanswered questions, this study investigated the short-term role of IAP and the sources of GBS transmission to newborns in the postnatal period.

\section{Methods}

This is a prospective, cohort study, with enrollment based on both maternal carrier status and infant GBS colonization. The current study was conducted at the
From the ${ }^{1}$ Neonatal Intensive Care Unit, Polyclinic University Hospital, Modena, Italy; ${ }^{2}$ Department of Infectious, Parasitic, and Immune-Mediated Diseases, Bacterial, Respiratory, and Systemic Disease Unit, Istituto Superiore di Sanità, Rome, Italy; ${ }^{3}$ Neonatal Intensive Care Unit, Hospital for Sick, Rimini, Italy; ${ }^{4}$ Biomedical Campus University, Rome, Italy; and

${ }^{5}$ Microbiology and Virology Unit, Polyclinic University Hospital, Modena, Italy

Funded by the European Community's Seventh Framework Program (FP7/2007-2013; HEALTH-F7-2007200481 Design of a Vaccine to Immunize Neonates against GBS Infections) and the Italian Ministry of Health (7M32). The authors declare no conflicts of interest.

0022-3476/\$ - see front matter. Copyright (C) 2013 Mosby Inc. All rights reserved. http://dx.doi.org/10.1016/j.jpeds.2013.05.064 
nursery of a tertiary care center with approximately 3500 live births/y and a screening-based strategy. According to Centers for Disease Control and Prevention guidelines, ${ }^{2}$ low vaginal and rectal swabs are taken at 35-37 weeks of gestation. Specimens are incubated on enriched culture medium testing. Penicillin (the recommended agent for IAP) ${ }^{2,3}$ is not produced in Italy. A standard dose of ampicillin ( $2 \mathrm{~g}$ intravenously plus $1 \mathrm{~g}$ intravenously every 4 hours until delivery) is given during active labor to GBS-colonized women or to women with risk factors for EOD and unknown carrier status. $^{13}$

Most women ( $>85 \%)$ with a positive screening at this center are given IAP ( $\geq 4$ hours $50 \%$ of cases). ${ }^{13}$ Protocol violation and/or precipitous delivery are the main reasons why IAP is not given. Written informed consent was obtained from participants. The project was approved by the local ethical committee (Prot. $\mathrm{N}^{\circ} 3357$ ). Enrollment began July 20,2008, and follow-up was completed on June 1, 2010. During the study period, there were 6864 parturient women.

At this center, approximately $20 \%$ of women are identified as positive GBS carriers. GBS noncarriers were enrolled according to a 1:2 ratio compared with GBS carriers.

Because of the imbalance in the frequency of available patient samples, maternal positive carriers were selected twice a week (Monday and Tuesday), but women who did not receive IAP were enrolled wherever possible.

Maternal eligibility included: (1) a singleton pregnancy; (2) antenatal screening at 35-37 weeks gestation (or GBS bacteriuria during pregnancy or a previous infant with GBS disease); (3) a residential address within $15 \mathrm{~km}$ of the hospital; and (4) sufficient communication skills in the national language (as evaluated by the investigating doctor).

Neonates room in with their mothers in the obstetric unit, unless special or intensive care is required. The obstetric unit consists of 4 single-bed rooms, 21 double-bed rooms, 1 triple-bed room, and 1 quadruple-bed room. Midwifery staff obtained both maternal and neonate specimens.

Maternal specimens were obtained from breast milk and the rectovaginal sites at discharge from hospital and at preorganized follow-up visits $(15,30$, and 60 days postpartum). Milk samples were collected by manual expression after washing both the breast and the nipple with soap, followed by saline solution cleansing. After discarding the initial drops, samples were collected in a sterile container.

Neonatal specimens were collected twice during hospitalization (throat, ear, and rectum within 10-24 hours of birth; throat and rectum 48-72 hours after birth/nursery discharge) and 3 times (15, 30, and 60 days) following hospital discharge.

Any newborns requiring empirical antibiotics were cultured prior to therapy initiation. Mothers were classified (according to the GBS carrier status at discharge from hospital) as follows: positive carriers (women confirmed culturepositive); negative carriers (women culture-negative at discharge from hospital, culture-positive at least once [at screening and/or at follow-up visits]); and noncarriers (women culture-negative throughout the study). GBS transmission to neonate was classified as immediately vertical (culture-positive newborns identified $\leq 24$ hours of birth); undetermined (no growth of GBS within 24 hours of birth, GBS identified at hospital discharge); and delayed (culturepositive newborns identified only after hospital discharge). In addition, neonatal colonization was categorized as heavy (GBS identified in all anatomic sites, at least once) or light (GBS identified in 1 anatomic site only).

Samples were collected using cotton-tipped swabs. The Cultiplast Transport System (Stuart transport medium without charcoal; LP Italiana, Milan, Italy) was used for transportation to the laboratory. If transportation or processing was delayed, specimens were stored at $4^{\circ} \mathrm{C}$. Maternal rectovaginal and neonatal rectal swabs were transferred $\leq 24$ hours to a $10 \mathrm{~mL}$ selective enrichment Todd-Hewitt broth (THB; bioMerieux Italia, Firenze, Italy) containing colistin $(10 \mathrm{mg} / \mathrm{L})$ and nalidixic acid $(15 \mathrm{mg} / \mathrm{L})$ and incubated for $18-24$ hours at $37^{\circ} \mathrm{C}^{2}$ The THB broth was subcultured on chromogenic culture media for identification of GBS (chromID StreptoB; bioMerieux Italia) and incubated in aerobic conditions for 24 hours. Neonatal throat and ear swabs were cultured on chromogenic culture media for identification of GBS (chromID StreptoB; bioMerieux Italia) without THB selective enrichment broth. Cultures were examined for colonies; pink or red colonies were identified using latex agglutination and standard biochemical tests ( Vitek2 colorimetric card for identification of gram positive bacteria; bioMerieux). Blood cultures were analyzed with an automated system (Bactec 9240; Becton-Dickinson, Milan, Italy).

Molecular typing of GBS was performed in 30 of 32 mother-baby pairs (not available for 1 case of immediate vertical and 1 case of delayed transmission). All strains were analyzed for serotype by using both a latex agglutination test (Statens Serum Institut, Copenhagen, Denmark) and a multiplex polymerase chain reaction assay; clonal relatedness was assayed using the pulsed field gel electrophoresis (PFGE) method, as already described. ${ }^{14}$ In particular, isolates with indistinguishable profiles were assigned to the same PFGE type and subtype; if PFGE profiles differed by up to 5 bands, isolates were considered as possibly related and identified as different subtype with the same PFGE type. Isolates with more than 5 bands of difference were considered unrelated and were identified as different PFGE types.

Standardized forms were used to collect both maternal and neonatal data. Statistical analysis was performed according to the $\chi^{2}$ test and ANOVA when appropriate. A $P$ value of $<.05$ was used as a threshold for statistical significance.

\section{Results}

Of the 182 mother-baby pairs recruited, 12 were lost to follow-up and 10 had missing data. Cultures from 160 mother-baby pairs were included in this study. 
Table I. Characteristics of the 160 mothers, according to group assignment based on culture status at hospital discharge

\begin{tabular}{|c|c|c|c|}
\hline & $\begin{array}{l}\text { Culture-positive } \\
\text { carriers } \\
(\mathbf{n}=\mathbf{8 3})\end{array}$ & $\begin{array}{l}\text { Culture-negative } \\
\text { carriers } \\
(n=26)\end{array}$ & $\begin{array}{c}\text { Non } \\
\text { carriers } \\
(n=51)\end{array}$ \\
\hline $\begin{array}{l}\text { Maternal age, } y \pm S D \\
\quad \text { (range) }\end{array}$ & $\begin{array}{l}33.9 \pm 4.9 \\
(18-44)\end{array}$ & $\begin{array}{l}33.4 \pm 4.2 \\
(23-42)\end{array}$ & $\begin{array}{c}32.8 \pm 4.6 \\
(22-40)\end{array}$ \\
\hline $\begin{array}{l}\text { Gestational age, mean } \\
\text { wk (range) }\end{array}$ & $\begin{array}{c}39.3 \\
(36-41)\end{array}$ & $\begin{array}{c}39.3 \\
(35-41)\end{array}$ & $\begin{array}{c}39.2 \\
(35-41)\end{array}$ \\
\hline $\begin{array}{l}\text { At least } 1 \text { risk factor for } \\
\text { EOD, } n^{\star}\end{array}$ & 9 & 6 & 5 \\
\hline Vaginal delivery, $\mathrm{n}$ & 71 & 23 & 44 \\
\hline $\begin{array}{l}\text { Positive culture results/ } \\
\text { antenatal screening, } \mathrm{n}^{\dagger}\end{array}$ & $74 / 77$ & $20 / 22$ & $0 / 51$ \\
\hline IAP administration, $\mathrm{n}(\%)^{\ddagger}$ & $57(68.7)$ & $20(74.1)$ & $3(5.9)^{\S}$ \\
\hline $\begin{array}{l}\mid A P \geq 4 \text { h prior to } \\
\text { delivery, } n\end{array}$ & 27 & 11 & 2 \\
\hline
\end{tabular}

*Risk factors were prolonged membrane rupture $(n=9)$ : 5 culture-positive-carriers, 2 culturenegative carriers and 2 noncarriers; GBS bacteriuria $(n=8): 6$ culture-positive carriers and 2 culture-negative carriers; preterm delivery $(n=6): 1$ culture-positive carrier, 2 culture-negative carriers and 2 noncarriers; previous infant with GBS disease $(n=2): 2$ culture-negative carriers maternal temperature $\left(\geq 38^{\circ} \mathrm{C}\right)(\mathrm{n}=1$, culture-positive carrier); and more than 1 risk factor was identified in 4 women (2 culture-positive carriers, 1 culture-negative carrier and 1 noncarrier). tWomen with GBS bacteriuria or with a previous infant with GBS disease were unscreened. $\ddagger$ Clyndamycin was administered to 2 women, and ampicillin to the remaining 78 women. $\S$ Because of a protocol violation, 3 women were treated unnecessarily with IAP (2 had prolonged membrane rupture $>18$ hours and 1 had no indication).

\section{Mothers}

Table I shows characteristics of the 160 mothers $(50 \%$ received IAP) according to group assignment; 153 were white European, 4 were black, and 3 were white African. Women are stratified (Figure 1) according to antenatal screening cultures and group assignment. Of the 94 women with culture-positive screen, $70(74.5 \%)$ were given IAP. Despite IAP administration, 54 of 70 (77.1\%) were identified as culture-positive carriers at hospital discharge.
Of the 56 women with culture-negative screen, 5 (8.9\%) were colonized; 3 at hospital discharge and 2 at a follow-up visit.

Table II displays the number of positive maternal rectovaginal culture results from screening to discharge, and at the 3 follow-up intervals. Sixty-two of 109 carrier women $(56.9 \%)$ were colonized at all visits. Rates of positive cultures at screening and post-delivery were compared. No significant difference was found between women who did or did not receive IAP (data not shown).

Of the 26 culture-negative carriers at hospital discharge, 20 (76.9\%) had received IAP. Among these 20 women, one-half were no longer colonized throughout the follow-up period and the other one-half were colonized at least once. Throughout culture testing, GBS was evidenced most strongly in the rectovaginal site $(n=423)$, compared with milk cultures $(\mathrm{n}=6)$.

\section{Neonates}

Among 160 newborns Table III, 35 (21.9\%) were colonized; 18 of them $(51.4 \%)$ were identified as heavily colonized. GBS was identified in rectal $(n=77)$, throat $(n=53)$, or ear cultures $(\mathrm{n}=11)$.

Eighty-three neonates were born to culture positive mothers; 30 of 83 (36.1\%) newborn infants were colonized; among them, 16 (53.3\%) were heavily colonized. Neonates exposed to IAP were less likely to be colonized than those who were not given IAP $(26.3 \%$ vs $57.7 \%, P<.05)$. A trend toward delayed transmission (GBS free at hospital discharge and colonized later) was associated with IAP administration. Heavy colonization following hospital discharge was not influenced by IAP administration. Rates of neonatal colonization at each time point were not significantly influenced by adequate $(5 / 27,18.5 \%)$ or inadequate $(10 / 30,33.3 \%$;

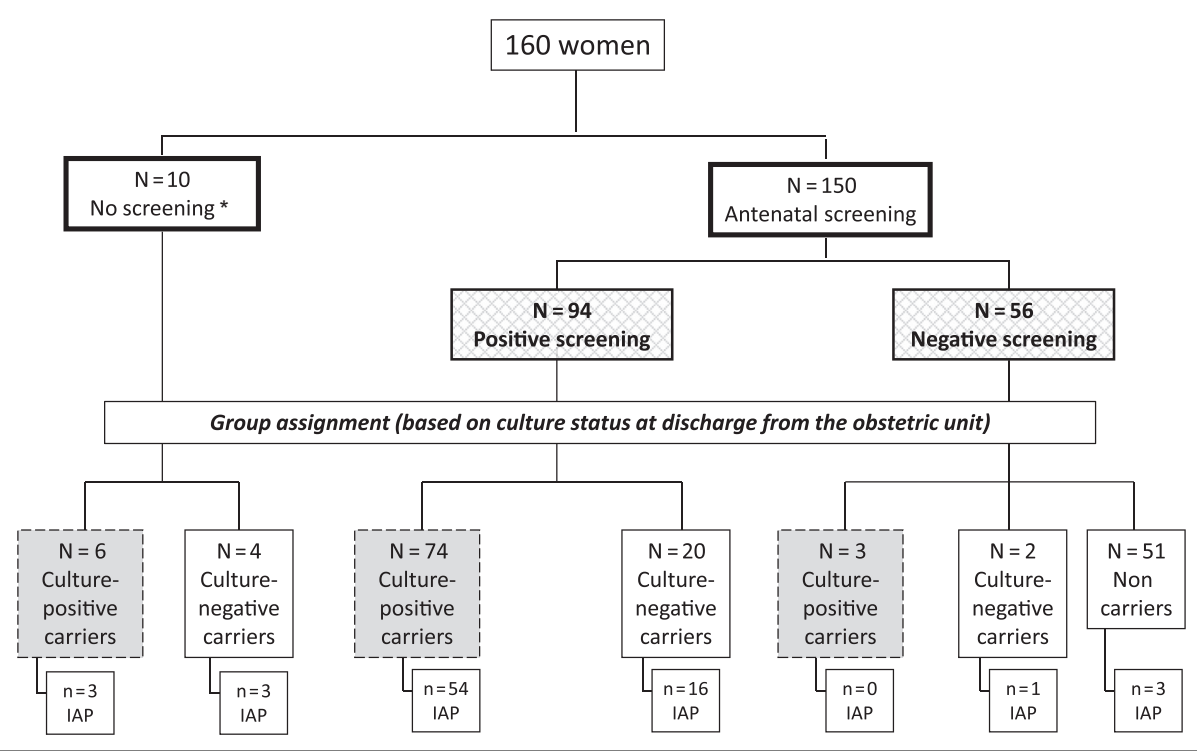

Figure 1. Antenatal screening, culture results, and group assignment in 160 mothers in the study population. * Eight had GBS bacteriuria, 2 had a previous infant with GBS disease. 
Table II. Rectovaginal culture positive results according to screening and follow-up visits

\begin{tabular}{|c|c|c|c|c|}
\hline \multirow[b]{2}{*}{ Culture testing } & \multicolumn{2}{|c|}{$\begin{array}{l}\text { Culture-positive } \\
\text { carriers } \\
(\mathrm{n}=\mathbf{8 3})\end{array}$} & \multicolumn{2}{|c|}{$\begin{array}{l}\text { Culture-negative } \\
\text { carriers } \\
(n=26)\end{array}$} \\
\hline & $\begin{array}{c}\text { No IAP } \\
(n=26)\end{array}$ & $\begin{array}{c}\text { IAP } \\
(n=57)\end{array}$ & $\begin{array}{l}\text { No IAP } \\
(\mathrm{n}=6) \\
\end{array}$ & $\begin{array}{c}\text { IAP } \\
(n=20)\end{array}$ \\
\hline At screening, $\mathrm{n}(\%)^{*}$ & $20(76.9)$ & $54(94.7)$ & $4(66.7)$ & $16(80.0)$ \\
\hline At hospital discharge, $\mathrm{n}(\%)$ & $26(100)$ & $57(100)$ & 0 & 0 \\
\hline \multicolumn{5}{|l|}{ At follow-up visits, $n(\%)$} \\
\hline $15 \mathrm{~d}$ & 24 (92.3) & $50(87.7)$ & $2(33.3)$ & $5(25.0)$ \\
\hline $30 \mathrm{~d}$ & $21(80.8)$ & $51(89.5)$ & $3(50.0)$ & $6(30.0)$ \\
\hline $60 \mathrm{~d}$ & 21(80.8) & $53(93.0)$ & $3(50.0)$ & $6(30.0)$ \\
\hline
\end{tabular}

${ }^{*}$ Ten women were not screened as they had GBS bacteriuria $(n=8)$ or a previous infant with GBS disease $(n=2)$.

$P=.33$ ) IAP administration. Longitudinal colonization (in hospital and following hospital discharge) for each motherbaby pair is shown in Figure 2 (available at www.jpeds.com).

There were 26 neonates born to culture negative mothers. Two $(7.7 \%)$ were colonized ( 1 case of heavy colonization). There was no case of immediate vertical transmission. Fifty-one neonates were born to noncarrier women; 3 $(5.9 \%) \ldots$ were colonized ( 1 case of heavy colonization). There was no case of immediate vertical transmission.

\section{Molecular Typing Analysis}

There were 32 colonized infants born to carrier mothers, but 2 neonatal specimens were missing. Isolates were available from 28 culture-positive carriers, 2 culture-negative carriers, and their respective babies. Molecular typing analysis of the 30 mother-baby pair available cultures, reported identified indistinguishable isolates (by both serotyping and PFGE analysis) for each pair. Each mother-baby pair maintained steadily the same serotype during time.

The most frequent serotype was III (14 cases), followed by V ( 8 cases), II ( 6 cases), Ia, and Ib ( 1 case, respectively). PFGE analysis distinguished 7 types (each associated with specific serotypes), and 20 subtypes. PFGE patterns were comparable with those previously identified in the same geographical area $^{14}$ and received the same PFGE type designation. New PFGE types (serotype II) were identified in 4 of the 6 strains (Table IV; available at www.jpeds.com). The rate of GBS transmission to the newborn was not affected by serotypes or by IAP administration.

Mothers of GBS-free neonates $(\mathrm{n}=77)$. Isolates were available from 68 mothers ( 9 were missing). Typing assays were successfully identified in 52 isolates $(76.5 \%)$. The most frequent serotype was type III (24 cases), followed by type Ia (13 cases), type V (9 cases), type Ib (3 cases), type II ( 2 cases), and type IX (1 case).

\section{Mastitis, Milk Cultures, and Neonatal Colonization}

Among 160 neonates, 14 (8.7\%) were exclusively formula fed and 146 (91.2\%) were partially or exclusively breastfed. One mother suffered from mastitis. Oral amoxicillin was given before milk was sampled, and the subsequent culture was found negative. GBS positive milk cultures were identified in 6 women, all culture-positive carriers. The respective neonates were all classified as heavily colonized, and 2 of them became infected (see below). Infected breast milk and neonatal colonization were diagnosed at the same followup visit; 3 out of 6 neonates without IAP exposure were colonized from nursery admission. Furthermore, 3 newborns were culture negative at admission but were identified as colonized at the first follow-up visit following hospital discharge; 2 were given IAP (which was inadequate in 1 case).

IAP exposure did not influence positive milk culture results (4/26 untreated mothers vs $2 / 57$ treated mothers, $P=.15)$. Milk bacterial count was available in 3 of the 6 cultures. Count was $10^{4} \mathrm{CFU} / \mathrm{mL}$ (1 case) or $10^{3} \mathrm{CFU} / \mathrm{mL}$ ( 2 cases).

\section{GBS Infections}

GBS infections were confirmed in 3 neonates. All were born to culture-positive mothers. Case one was born to a mother with membrane rupture (7 hours) who became pyrexial

Table III. Colonized newborns and transmission modes by maternal colonization status

\begin{tabular}{|c|c|c|c|c|c|c|}
\hline & \multicolumn{2}{|c|}{$\begin{array}{l}\text { Culture-positive carriers } \\
(n=83)\end{array}$} & \multicolumn{2}{|c|}{$\begin{array}{l}\text { Culture-negative carriers } \\
\qquad(\mathrm{n}=26)\end{array}$} & \multicolumn{2}{|c|}{$\begin{array}{l}\text { Noncarriers } \\
(\mathrm{n}=51)\end{array}$} \\
\hline & $\begin{array}{l}\text { No IAP } \\
(n=26)\end{array}$ & $\begin{array}{c}\text { IAP } \\
(n=57)\end{array}$ & $\begin{array}{l}\text { No IAP } \\
(\mathrm{n}=6)\end{array}$ & $\begin{array}{c}\text { IAP } \\
(n=20)\end{array}$ & $\begin{array}{l}\text { No IAP } \\
(n=48)\end{array}$ & $\begin{array}{c}\text { IAP } \\
(n=3)\end{array}$ \\
\hline Total colonized newborns, $\mathrm{n}(\%)$ & $15(57.7)$ & $15(26.3)^{\dagger}$ & $1(16.7)$ & $1(5.0)$ & $2(4.2)$ & 1 (33.3) \\
\hline During hospitalization & $14(53.8)$ & $3(5.3)^{\star}$ & $1(16.7)$ & 0 & 1 & 0 \\
\hline Following hospital discharge & $13(50.0)$ & $12(21.1)^{\dagger}$ & $1(16.7)$ & $1(5.0)$ & $2(4.2)$ & $1(33.3)$ \\
\hline Heavily colonized newborns, $\mathrm{n}(\%)^{\S}$ & $9(34.6)$ & $7(12.3)^{\dagger}$ & $1(16.7)$ & 0 & 0 & $1(33.3)$ \\
\hline During hospitalization & $7(26.9)$ & $1(1.8)^{*}$ & $1(16.7)$ & 0 & 0 & 0 \\
\hline Following hospital discharge & $7(26.9)$ & $6(10.5)$ & 0 & 0 & 0 & $1(33.3)$ \\
\hline \multicolumn{7}{|l|}{ Modes of transmission, $\mathrm{n}(\%)^{\S}$} \\
\hline Immediate vertical & $12(46.2)$ & $2(3.5)^{\star}$ & 0 & 0 & 0 & 0 \\
\hline Undetermined & $2(7.7)$ & $1(1.8)$ & $1(16.7)$ & 0 & $1(2.1)$ & 0 \\
\hline Delayed & $1(3.8)$ & $12(21.1)^{ \pm, \pi}$ & 0 & $1(5.0)$ & $1(2.1)$ & $1(33.3)$ \\
\hline
\end{tabular}

${ }^{\star} P<.01$.

$+P<.05$.

$\ddagger P<.1$.

$\S$ Heavily colonized newborns and modes of transmission are calculated as percentage of total neonates

IAmong 12 neonates with IAP administration (culture negative during the hospitalization), delayed transmission was confirmed following hospital discharge (at 15 days, 10 cases; or at 30 days, 2 cases). 
$\left(39^{\circ} \mathrm{C}\right) 2$ hours before admission. Intrapartum ampicillin was given 3 hours before delivery. The baby was ill at birth (clinical signs of sepsis, positive surface cultures, abnormal laboratory testing, negative blood, and cerebrospinal fluid cultures) and recovered following a 10 days course of antibiotics. The neonate was found no longer colonized at subsequent follow-up visits. Respective milk cultures were sterile.

Case 2 (no EOD risk factors, intact membrane, no IAP given) presented with early-onset clinical sepsis 20 hours after birth and was treated with a 10-day course of ampicillin intramuscularly. At 18 days, the baby was again treated with a second course of antibiotics because of a GBS urinary tract infection. The neonate was heavily colonized from birth through the last follow-up visit; milk culture was positive at discharge from hospital but sterile at the following follow-up visits.

Case three (no EOD risk factors, 3 hours membrane rupture) received adequate IAP (ampicillin, 3 doses). The baby was culture-negative at birth and became heavily colonized at the first follow-up visit ( 15 days old), when milk culture was found to be positive. Milk was sterile at the following follow-up visits. GBS urinary tract infection was diagnosed at age 35 days, at which time the newborn received a 7-day course of amoxicillin orally. The baby was not colonized at the last follow-up visit.

\section{Discussion}

The modes of GBS transmission to the newborn infant are unclear. ${ }^{15,16}$ Studies performed prior to the introduction of IAP were inconclusive in determining the predominant sources (nosocomial, community, or maternal) of postnatal transmission. ${ }^{7-9,17-24}$ Furthermore, most of these studies had important limitations, such as suboptimal culture methods, improper sites of culture collection, or insufficient number and frequency of postdelivery sampling. ${ }^{8,9,18-20,25}$ From these studies, neonatal colonization was proven to be acquired predominately during the delivery process, ${ }^{8,9,18,20,25}$ and the infants at the highest risk of GBS colonization were identified as those born to untreated, carrier women. ${ }^{13,24,26,27}$

In the current study, neonates born to culture-positive carriers exposed to IAP had lower rates of colonization (26.3\%) compared with neonates born to culture-positive carriers without IAP exposure (57.7\%), and they also had lower rates of heavy colonization $(12.3 \%$ vs $34.6 \%)$.

Heavy neonatal colonization and delayed transmission (evident in most cases at the first follow-up visit) were more common in infants born to culture-positive carrier women, possibly because their mothers were more heavily colonized than culture-negative carriers.

No significant difference was found in rates of positive postdelivery cultures between women treated with IAP or untreated. GBS may persist for many weeks following antibiotic therapy, ${ }^{28}$ and hence, mothers remain an important source of transmission. ${ }^{29}$ Indeed, GBS strains cultured from both neonates with delayed transmission and their respective mothers showed the same serotype and indistinguishable PFGE profiles, with all bands matching closely within each GBS pair, indicating the presence of the same strains in all motherbaby pair cases. We hypothesize that GBS may have been transmitted from the mother at home when no longer suppressed by IAP.

Reported rates of GBS transmission to neonates of noncarrier women range from $1.2 \%$ to $33 \%-65 \%{ }^{8,20,24}$ This discrepancy may in part be explained by inattention to hygiene practices, especially hand washing, of the caregivers during the infants' hospitalization. In this study, GBS transmission from nonmaternal sources was confirmed in only 3 cases $(5.9 \%)$. Cross-transmission within the obstetric ward was unlikely, as most infants exposed to IAP were culture negative during hospitalization, and most women were hospitalized in single or double rooms, with individual maternal-infant nursing.

Case reports have implicated breast milk as a possible source of LOD, ${ }^{10-12}$ but the relevance of breast feeding is unknown. Kubin ${ }^{30}$ reported 3.5\% GBS infected milk samples, but most infants feeding from the infected breast milk remained healthy. However, the results by Kubin et al should be interpreted with caution, as the data regarding GBS rectovaginal carriage, IAP administration, and neonatal colonization rates were not provided.

In the current study, a few culture-positive carrier women had culture-positive milk. Most heavily colonized neonates were born to culture-positive carrier mothers, and the authors suggest that the milk may have been contaminated from the throat of the healthy, heavily colonized newborns. Two out of 3 newborn infants with GBS infection were fed culture-positive milk, but there was no evidence that the milk was the cause of the neonatal infections. One of them (case 2) became ill long before (30 hours) the milk was found to be positive.

This study has a number of limitations. The relatively small sample size may preclude any detailed analyses, especially with regard to differences in transmission by serotype. In addition, maternal cultures were obtained after delivery, and therefore, IAP administration may have affected the cultures. However, our previous unpublished data show that maternal cultures, taken $\geq 48$ hours postdelivery, quite closely reflect GBS status, independent of IAP. Finally, IAP exposure may underestimate the true GBS transmission as culture-negative results at hospital discharge may be falsely negative. However, this study shows that neonates exposed to IAP were more likely to remain culture-negative following hospital discharge, when the effect of the antibiotics should no longer inhibit GBS growth. IAP may be effective in interrupting vertical transmission.

Further studies should determine whether infection control measures during hospitalization and careful hygiene in infant nursing reduce the risk of LOD. Studies should also include culture specimens from other family members, as important information regarding community transmission could be identified. 
The authors thank Johanna Chester for her critical revision and editorial assistance, Marco Pataracchia e Giovanna Alfarone for his excellent technical expertise in microbiological analysis of the strains, and Professor Carol J. Baker for revising the initial study design and gave valuable suggestions.

Submitted for publication Jan 28, 2013; last revision received Apr 18, 2013; accepted May 31, 2013.

Reprint requests: Alberto Berardi, MD, Unità Operativa di Terapia Intensiva Neonatale, Azienda Ospedaliero-Universitaria Policlinico, Via del Pozzo, 71, 41100 Modena, Italy. E-mail: berardi.alberto@policlinico.mo.it

\section{References}

1. Edwards MS, Baker CJ. Group B Streptococcal infections. In: Remington JS, Klein JO, eds. Infectious diseases of the fetus and newborn infant. 5th ed. Philadelphia: Saunders; 2005. p. 1091-156.

2. Schrag S, Gorwitz R, Fultz-Butts K, Schuchat A. Prevention of perinatal group B streptococcal disease. Revised guidelines from CDC. MMWR Recomm Rep 2002;51(RR-11):1-22.

3. Verani JR, McGee L, Schrag SJ, Division of Bacterial Diseases, National Center for Immunization and Respiratory Diseases, Centers for Disease Control and Prevention (CDC). Prevention of perinatal group B streptococcal disease-revised guidelines from CDC, 2010. MMWR Recomm Rep 2010;59(RR-10):1-36.

4. Berardi A, Lugli L, Baronciani D, Rossi C, Ciccia M, Creti R, et al. Group B Streptococcus early-onset disease in Emilia-Romagna: review after introduction of a screening-based approach. Pediatr Infect Dis J 2010; 29:115-21.

5. Lin FY, Weisman LE, Troendle J, Adams K. Prematurity is the major risk factor for late-onset group B Streptococcus disease. J Infect Dis 2003;188: 267-71.

6. Dillon HC, Khare S, Gray BM. Group B streptococcal carriage and disease: a 6-year prospective study. J Pediatr 1987;110:31-6.

7. Steere AC, Aber RC, Warford LR, Murphy KE, Feeley JC, Hayes PS, et al. Possible nosocomial transmission of group B Streptococci in a newborn nursery. J Pediatr 1975;87:784-7.

8. Paredes A, Wong P, Mason EO Jr, Taber LH, Barrett FF. Nosocomial transmission of group B Streptococci in a newborn nursery. Pediatrics 1977;59:679-82.

9. Anthony BF, Okada DM, Hobel CJ. Epidemiology of the group B Streptococcus: maternal and nosocomial sources for infant acquisitions. J Pediatr 1979;95:431-6.

10. Bingen E, Denamur E, Lambert-Zechovsky N, Aujard Y, Brahimi N, Geslin P, et al. Analysis of DNA restriction fragment length polymorphism extends the evidence for breast milk transmission in Streptococcus agalactiae late-onset neonatal infection. J Infect Dis 1992;165: 569-73.

11. Dinger J, Müller D, Pargac N, Schwarze R. Breast milk transmission of group B streptococcal infection. Pediatr Infect Dis J 2002;21:567-8.

12. Kotiw M, Zhang GW, Daggard G, Reiss-Levy E, Tapsall JW, Numa A. Late-onset and recurrent neonatal group B streptococcal disease associated with breast-milk transmission. Pediatr Dev Pathol 2003;6:251-6.
13. Berardi A, Rossi C, Biasini A, Minniti S, Venturelli C, Ferrari F, et al. Efficacy of intrapartum chemoprophylaxis less than 4 hours duration. J Matern Fetal Neonatal Med 2010;23:1456-60.

14. Imperi M, Gherardi G, Berardi A, Baldassarri L, Pataracchia M, Dicuonzo G, et al. Invasive neonatal GBS infections from an area-based surveillance study in Italy. Clin Microbiol Infect 2011;17:1834-9.

15. Berardi A, Tzialla C, Riva M, Cerbo RM, Creti R. Group B Streptococcus: early- and late-onset infections. J Chemother 2007;19(Suppl 2):24-7.

16. Jordan HT, Farley MM, Craig A, Mohle-Boetani J, Harrison LH, Petit S, et al. Revisiting the need for vaccine prevention of late-onset neonatal group B streptococcal disease: a multistate, population-based analysis. Pediatr Infect Dis J 2008;27:1057-64.

17. Baker CJ, Barrett FF. Transmission of group B Streptococci among parturient women and their neonates. J Pediatr 1973;83:919-25.

18. Ferrieri P, Cleary PP, Seeds AE. Epidemiology of group B streptococcal carriage in pregnant women and newborn infants. J Med Microbiol 1977;10:103-7.

19. Pass MA, Gray MB, Khare S, Dillon HC. Prospective studies of group B streptococcal infections in infants. J Pediatr 1979;95:437-43.

20. Yow MD, Leeds LJ, Thompson PK, Mason EO Jr, Clark DJ, Beachler CW. The natural history of group B streptococcal colonization in the pregnant woman and her offspring. I. Colonization studies. Am J Obstet Gynecol 1980;137:34-8.

21. Gardner SE, Mason EO, Yow MD. Community acquisition of group B Streptococcus by infants of colonized mothers. Pediatrics 1980;66:873-5.

22. Boyer KM, Vogel LC, Gotoff SP, Gadzala CA, Stringer J, Maxted WR. Nosocomial transmission of bacteriophage type 7/11/12 group B Streptococci in a special care nursery. Am J Dis Child 1980;134:964-6.

23. Band JD, Clegg HW II, Hayes PS, Facklam RR, Stringer J, Dixon RE. Transmission of group B Streptococci. Traced by use of multiple epidemiologic markers. Am J Dis Child 1981;135:355-8.

24. Boyer KM, Gadzala CA, Kelly P, Gotoff SP. Selective intrapartum chemoprophylaxis of neonatal group B streptococcal early-onset disease. III. Interruption of mother-to-infant transmission. J Infect Dis 1983;148: 810-6.

25. Easmon CS, Hastings MJ, Clare AJ, Bloxham B, Marwood R, Rivers RP, et al. Nosocomial transmission of group B Streptococci. Br Med J (Clin Res Ed) 1981;283:459-61.

26. Easmon CS, Hastings MJ, Blowers A, Bloxham B, Deeley J, Marwood R, et al. Epidemiology of group B Streptococci: one year's experience in an obstetric and special care baby unit. Br J Obstet Gynaecol 1983;90: 241-6.

27. De Cueto M, Sanchez MJ, Sampedro A, Miranda JA, Herruzo AJ, RosaFraile M. Timing of intrapartum ampicillin and prevention of vertical transmission of group B Streptococcus. Obstet Gynecol 1998;91:112-4.

28. Gardner SE, Yow MD, Leeds LJ, Thompson PK, Mason EO Jr, Clark DJ. Failure of penicillin to eradicate group B streptococcal colonization in the pregnant woman. A couple study. Am J Obstet Gynecol 1979;135: 1062-5.

29. Berardi A, Rossi C, Lugli L, Creti R, Bacchi Reggiani ML, Lanari M, et al. Group B Streptococcus late-onset disease: 2003-2010. Pediatrics 2013; 131:e361-8.

30. Kubín V, Mrastíková H, Paulová M, Motlová J, Franěk J. Group B Streptococci in the milk of lactating mothers. Zentralbl Bakteriol Mikrobiol Hyg A 1987;265:210-7. 
Table IV. Microbiological characteristics of bacterial isolates from 30 mother-baby pairs

\begin{tabular}{lllc}
\hline & Serotype & n/total cases & PFGE types (n) \\
\hline Culture-positive carriers & Ia & $1 / 28$ & Undetermined \\
& Ib & $1 / 28$ & 1 \\
& II & $6 / 28$ & $5^{\star}$ \\
& III & $12 / 28$ & 10 \\
Culture-negative carriers & V & $8 / 28$ & 3 \\
& III & $2 / 2$ & 2 \\
\hline
\end{tabular}

*With respect to PFGE types previously identified, ${ }^{14} 3$ out of 5 types were newly identified.

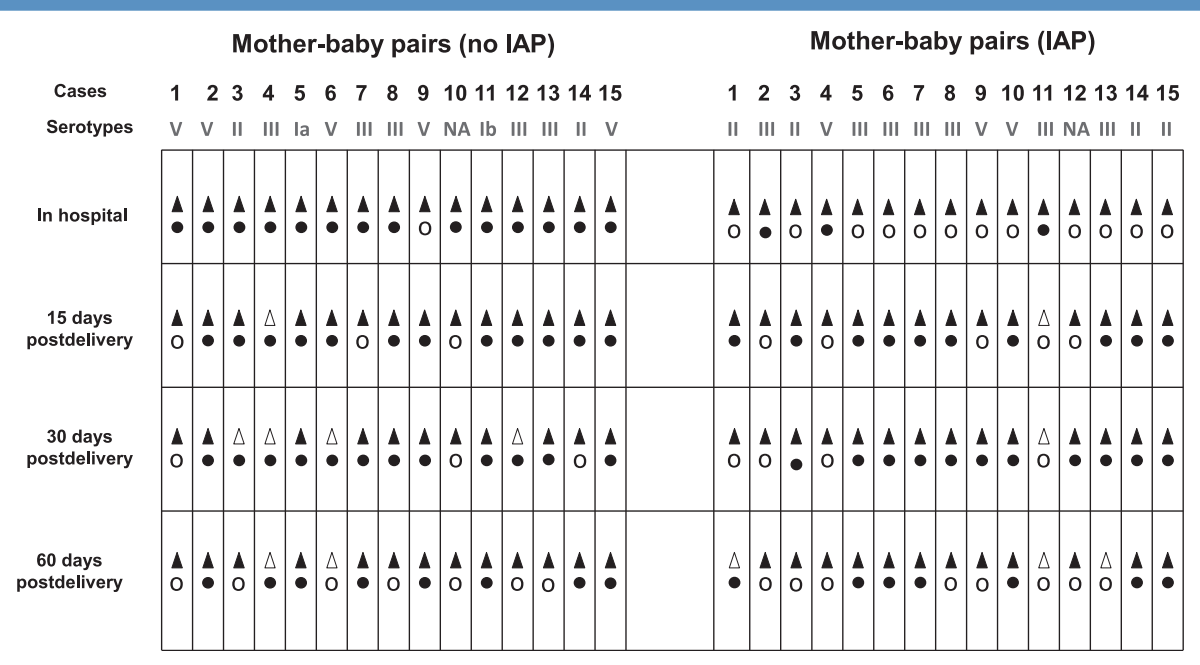

Figure 2. Colonization and serotypes among 30 culture-positive carrier mothers and their infants (in hospital and at follow-up visits). The right side of the Figure shows cases with IAP administration (cases 1, 4, 6, 10, 13, and 14 were given adequate IAP). $N A$, not assessed. $\Delta$ Colonized mother. $\Delta$ Not colonized mother. Colonized newborn. $\bigcirc$ Not colonized newborn. 